\title{
DEGRADABLE POLYMERIC MATERIALS FOR OSTEOSYNTHESIS: TUTORIAL
}

\author{
D. Eglin* and M. Alini
}

Biomaterials and Tissue Engineering Program, AO Research Institute, CH-7270 Davos, Switzerland

\begin{abstract}
This report summarizes the state of the art and recent developments and advances in the use of degradable polymers devices for osteosynthesis. The current generation of biodegradable polymeric implants for bone repair utilising designs copied from metal implants, originates from the concept that devices should be supportive and as "inert" substitute to bone tissue. Today degradable polymeric devices for osteosynthesis are successful in low or mild load bearing applications. However, the lack of carefully controlled randomized prospective trials that document their efficacy in treating a particular fracture pattern is still an issue. Then, the choice between degradable and non-degradable devices must be carefully weighed and depends on many factors such as the patient age and condition, the type of fracture, the risk of infection, etc. The improvement of the biodegradable devices mechanical properties and their degradation behaviour will have to be achieved to broaden their use. The next generation of biodegradable implants will probably see the implementation of the recent gained knowledge in cellmaterial interactions and cells therapy, with a better control of the spatial and temporal interfaces between the material and the surrounding bone tissue.
\end{abstract}

Keywords: osteosynthesis, degradable, $\operatorname{poly}(\alpha-$ hydroxyacids).

*Address for correspondence:

D. Eglin

Biomaterials and Tissue Engineering Program

AO Research Institute, Clavadelerstrasse 8,

CH-7270 Davos,

Switzerland

Telephone Number: +41814142480

FAX Number: +41814142288

Email: david.eglin@aofoundation.org

\section{Introduction}

The development of materials for osteosynthesis has been co-continuous with the improvement of the bone fixation knowledge and the complexity of surgical procedures. Thus, thanks to their outstanding mechanical properties, metal devices made of titanium alloys are the gold standard for the majority of the fracture fixation treatments. However, they have several significant drawbacks. First, after the fracture healing, a second operation is often necessary to remove the implants especially in paediatric and this has several risks such as infection, removal problems of jammed implants, implants migration and associated extra health care costs. Secondly, metal devices cause magnetic resonance imaging artefacts leading to visualization difficulties to monitor tissue regeneration. Finally, the high modulus of elasticity of metals compared to bone, results in the implant retaining a large fraction of the mechanical load applied to the bone. This is known as the "stress shielding" effect which leads to bone resorption, implant loosening and, consequently, the need for a second operation.

As a consequence, the thinking for many years has been to use a material that will degrade and which gradually loss is strength at the same rate of bone healing, would improve the final outcome of fracture surgery. The quest for such a material was the initial driving force behind the research on degradable polymers for osteosynthesis.

\section{Biodegradable Materials}

Conventional osteosynthesis devices made of metals have been challenged and the use of degradable devices considered since the middle of the last century (Van der Elst et al., 2000). This can be linked to the discovery and the development of new materials such as poly(glycolic acid), a polymer that has been shown to be unstable in a "normal" environment.

\section{Definitions}

A biodegradable material can be defined as a material that breaks down in vivo, but with no proof of its elimination from the body (Vert et al., 1992). For example, biodegradable polymeric systems or devices can be attacked by a biological environment so that the integrity of the material is affected and produces degradation fragments. A material shows preferential surface erosion or bulk erosion depending of its intrinsic properties (water diffusion and degradation rate) and its size (Von Burkersroda et al., 2002). Such fragments can be carried away from their site of implantation but not necessarily from the body. A material is called bioresorbable when it shows degradation and further resorption in vivo. Thus, 
resorbable polymer by-products are eliminated through natural pathways either because of simple filtration or after their metabolization. Bioresorption is then a concept that reflects total elimination of the initial foreign material and any by-products (low molecular weight compounds). Thus, a biodegradable polymer is not necessarily bioresorbable. Finally, a material is bioabsorbable if it can dissolve in body fluids without any molecular degradation. The material is then excreted.

A material implanted to maintain the mechanical properties of a bone fracture during the healing period should, by definition, have adequate strength, wear and fatigue resistance. It should also be safe and easy to use. When the device is to be removed after healing, it is an advantage that the implant does not present intimate and significant bonding with the surrounding tissues. However, when considering a biodegradable implant a strong bonding between the device and the bone may improve the stability of the fracture. The material should then allow bone apposition to its surface. This property is called osteoconduction. An osteoinductive material does not only support bone formation but positively enhances the bone formation because of its surface topography, chemistry and the release of bioactive degradation by-products (LeGeros, 2002).

\section{Materials}

Biodegradable materials intended for bone repair can be from many origins, but they are all metabolized in the body without leaving traces after fulfilling their purpose. They should not be toxic or induce a negative response disproportionate to their beneficial effect. They should be easily processed into the desired shape, have an acceptable shelf-life and be easily sterilized. The degradable devices should possess adequate mechanical properties (e.g. strength, elasticity). These must gradually be lost during the degradation to progressively transfer mechanical loads to the newly forming bone (Simon et al., 1998).

There are many materials that have been considered as potential candidates for biodegradable implants: magnesium metal and alloys, calcium phosphate ceramics and glasses, and polymers (Bohner, 2000; Gogolewski, 2000; Staiger et al., 2006). The biodegradable polymers have probably generated the most expectation as degradable materials for osteosynthesis. Several polymers already have a long history as degradable materials for biomedical and pharmaceutical applications. Resorbable sutures, for example, have been commercialized for decades before biodegradable devices for osteosynthesis were available (Middleton and Tipton, 2000).

Biodegradable polymers. They are either of natural or synthetic origin. Natural polymers can closely mimic the biological environment (e.g. extracellular matrix) and present some biofunctionalities, however none has yet been processed and modified successfully into strong fixation devices (Bonzani et al., 2006). Synthetic polymers have the advantage to have a controlled and reproducible molecular structure and to be non-immunogenic. Biodegradable synthetic polymers have to be well tolerated upon implantation and during their degradation if considered for applications in contact with bone. It should elicit no or a minimal inflammation from the surrounding tissue and no reaction from remote locations. There are many factors common to any fixation device that can trigger a foreign body reaction: the implant geometry, the geometry and size of the implant, the implant surface properties, etc. The rate of implant degradation, the associated structural and surface property changes, and the biocompatibility of the released by-products are supplementary factors that have to be taken into account for these devices. The implant biocompatibility reflects the body's tolerance to these factors. Ideally, to avoid loosening, fluid accumulation and possible re-fracture, the implant must not induce bone resorption or the formation of a fibrous tissue encapsulation.

Polyesters. Poly( $\alpha$-hydroxyacids) are probably the most widely researched biodegradable synthetic polymers and they have been used in orthopaedics since the 1960s (Pitt, 1992; Vert, 1992; Middleton and Tipton; 2000, Van der Elst et al., 2000; Vert, 2005). Among the poly( $\alpha-$ hydroxyacids) or aliphatic polyesters; the most studied are poly(glycolic acid) PGA, poly(lactic acid) PLA and their co-polymers. These polymers have been FDA approved for several medical applications and the vast majority of the commercial biodegradable fixation devices are based on these. PGA polymer is made of glycolic acid repeating unit and PLA monomeric unit, lactic acid exists as two optical isomers, L and D-lactic acid. The natural occurring L-lactic acid is more often used to synthesize poly(L-lactic acid) PLLA. Polydioxanone PDS, a poly(ether-ester) and poly( $\varepsilon$-caprolactone) PCL are also widely studied polyester for medical applications. The polyesters can be produced by direct polycondensation of the monomers (e.g., lactic acid), but high temperatures are needed, resulting in a polymer with high polydispersity and low molecular weight. The ring-opening polymerization of cyclic monomer or dimer is preferred to obtain high molecular weight polyesters (Fig. 1).

In the presence of a catalyst, the ring-opening polymerization takes place at middle temperature leading to high molecular weight and low polydispersity polyesters suitable for biomedical applications. Also, inherent to the mechanism of polymerization and its sensitivity to moisture, the reproducibility and repeatability of the polyesters synthesis are difficult to achieve. This may lead to change of polymer properties from batch to batch and from one supplier to another. It is noteworthy that copolymers or ter-polymers which are polymers that contain two or more monomer types in their molecular chain can be produced by ring-opening polymerization of cyclic lactide, glycolide dimers and caproic monomers allowing the synthesis of polyesters with modified molecular structure, mechanical properties and degradation pattern (Glarner and Gogolewski, 2007). Bacterial polyesters or polyhydroxyalkanoates (e.g. poly( $\beta$-hydroxybutyrate)) are also of interest as they can be produced from renewable resources. In the body, they degrade in more than 12 months and they have shown favourable interactions with bone. However, their production and extraction are still extremely costly (Chandra and Rustgi, 1998). 
<smiles>CC1(C)OC(=O)C(C)(C)OC1=O</smiles>
$\underset{\text { Catalyst }}{\stackrel{\mathrm{ROP}}{\longrightarrow}}$<smiles>CC(C)(C)OC(=O)[Pb](C)(C)C</smiles>

poly(L-lactic acid)

chain length and ultimately for the size of the chains that diffuse into the surroundings, when small enough. Many factors affect the hydrolytic degradation process and its speed (Li et al., 1990; Vert, 2005):

The molecular composition of the polymer. The monomers that comprise the resorbable polymer affect the sensitivity of hydrolysable bonds. For example, PGA made of glycolic acid repeating units degrades faster than PCL made of caproic acid monomeric units. The polymer molecular weight (Mn). The length of the polymer chains influences the degradation rate. Obviously, the longer the polymer chain is (high Mn), the more hydrolytic chain scissions are necessary to obtain by-products which are able to diffuse out of the device. This reduces the degradation rate of the polymer.

The crystallinity. Crystallinity is a measure of the organization, packing and interactions in a material. Although a polymer cannot be $100 \%$ crystalline, a semi-crystalline polymer is well organized at molecular level and present many inter- and intra-molecular bonds (e.g. hydrogen bonds). In contrast, an amorphous polymer does not present a close packed organization. Therefore, small molecules such as water can more readily diffuse in amorphous polymers than in semicrystalline polymeric materials. The consequence is that amorphous polymers are hydrolyzed faster than semi-crystalline ones. polyurethanes have shown to be promising as membranes for guided tissue regeneration and as porous structures for filling bone defects (Gogolewski and Gorna, 2007).

\section{Polymer Degradation Mechanisms}

Bone is a chemically active environment composed of many molecules including water, salts, enzymes, free radicals, etc. All may theoretically affect polymers degradation. Degradable polymers intended for fixation devices degrade primarily as a result of chemical and enzymatic mechanisms. UV-light, irradiation and mechanical load have also a marked effect on the disappearance of some polymers (Chandra and Rustgi, 1998; Middleton and Tipton, 2000).

\section{Chemical degradation}

Most polymer devices degrade solely as a result of chemical degradation. The advantage of a polymer that degrades non-enzymatically by a chemical mechanism is that the kinetics of degradation can be controlled by the tailoring of the polymer. In addition, the assertion that PLLA and others synthetic polymers degrade exclusively by chemical mechanisms in vivo is disputable and it has been demonstrated that enzymes (proteinases) could also degrade PLLA (Williams, 1981).

Hydrolysis is the most frequent mechanism of synthetic polymer degradation in a biological environment. Hydrolysis is also the major mechanism of degradation for polyesters (Fig. 2) (Pitt et al., 1981; Li, 1999). Ester, ether, urethane and urea bonds have decreasing sensitivity to hydrolysis in the respective order. They are responsible for the polymer chain cleavage, the decrease of polymer

As an example, poly( $\alpha$-hydroxyacids) hydrolytic degradation is influenced by four major factors: The hydrolysis rate constant of the ester bond (composition); the diffusion coefficient of water in the matrix (crystallinity); the diffusion coefficient of the chain fragments within the polymeric matrix and the solubility of degradation products (Mn and crystallinity). The byproducts produced by the hydrolysis of ester bonds in poly $(\alpha$-hydroxyacids $)$ are acidic. The consequence is that polymeric devices, made of PLLA or PGA degrade faster in the presence of the acidic by-products. This autocatalytic phenomenon, which is observed in massive samples only (above few millimetres), is due to the newly formed degradation products and the differential diffusion rate between the surface and the bulk of the material. Chiefly, the acidic by-products accumulate into the implant centre and lower the $\mathrm{pH}$ in the bulk of the material. The lower $\mathrm{pH}$ increases the speed of hydrolytic scission in the centre in comparison to the surface. This phenomenon has been observed in early polyester devices and was accompanied with a burst of acidic products released in the surrounding and a dramatic loss in mechanical properties that may have been responsible for the observation of delayed inflammatory response and mechanical failure respectively (Bostman and Pihlajamaki, 2000) (Fig. 2).

In the body, the L-lactic released by PLLA is recycled by conversion into glycogen in the liver or incorporated into the tricarboxylic acid cycle and excreted in the lungs as carbon dioxide and water. Glycolic acid can be excreted in the urine or also enter the tricarboxylic acid cycle as Llactic acid. Others polyesters and resorbable polymer byproducts follow similar biological pathways (Simon et al., 


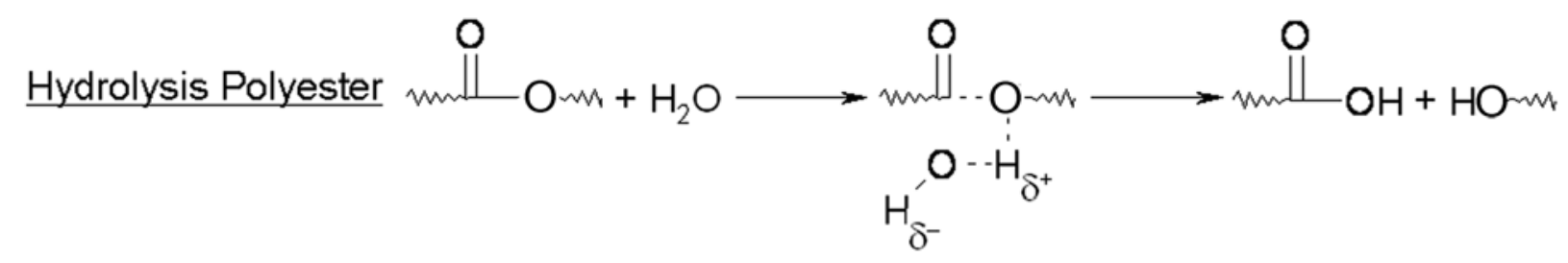

Devices Degradation

Bulk
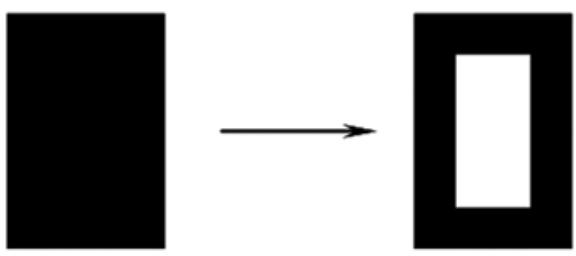

Erosion
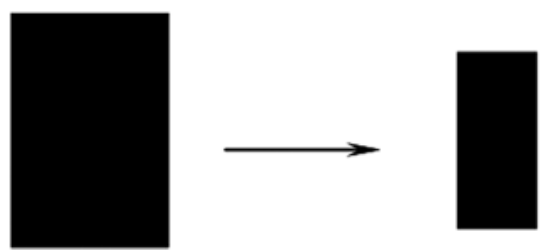

Figure 2. Schematics of the hydrolysis mechanism of polyester and of the bulk and surface erosion of degradable polymeric devices.

1998). Finally, the presence of impurities, fillers, plasticizers and additives can all influence the hydrolysis of polymers by modifying the crystallinity. They can also have a role in the polymer degradation by inducing oxidation reaction. Oxidation is another mechanism of polymer degradation for polyethers and polyurethanes. There are actually several oxidation mechanisms including oxidation by free radical and metals ions. For polyester devices, this is important mostly when considering shelflife of devices and sterilization as oxidation could occur during exposure to light (UV) and high energy radiation sterilization processes. Finally, polymer oxidation can also be performed by enzymes.

\section{Enzymatic degradation}

Enzymatic degradation occurs to different extents depending on the polymer type. Enzymatic degradation encompasses proteolytic and glycolytic degradation pathways. Macromolecules present in human tissues such as collagen, fibrinogen and hyaluronan are obviously susceptible to enzymatic degradation in vivo (Chandra and Rustgi, 1998). Synthetic polymers can also be degraded enzymatically: First, by enzymes released by phagocytes, macrophages and neutrophils present in a wound and which endeavour to digest the foreign implanted material. This living tissue reaction that takes place in an injury site always occurs, even in the most favourable case (biocompatible material), upon the implantation of foreign device. Most polymeric devices are seldom degraded by this enzymatic degradation. Also, it has long been recognized that in vivo, poly( $\alpha$-hydroxyacids $)$ degrade faster than in vitro, and the site of implantation and its vascularisation influence the rate of degradation. Secondly, a specifically designed enzyme sensitive segment introduced into the polymeric chains (e.g. polyurethane) can make the polymer sensitive to a specific enzyme (Rockwood et al., 2007). This was primarly developed for drug delivery systems, in which the active molecule encapsulated in the polymer is released upon enzymatic degradation, and for the synthesis of biodegradable hydrogels for bone tissue engineering applications. The advantages of the enzymatic over purely chemical degradation are the efficiency and the selectivity of the mechanism.

\section{Biodegradable Polyester Devices}

\section{Processing}

Mechanical properties of major biodegradable polymers, bone and others materials are reported in Table 1.

Machining, compression moulding, melt spinning and hot drawing are the most common processing methods for polymers. They all affect the intrinsic properties of the polymer to some degree and as a result the device properties (e.g. degradation and mechanics) (Table 1). For example, during the melting processing of PLLA, the heat and the presence of moisture reduce the polymer molecular weight (Simon et al., 1998). The consequence is a decrease of mechanical properties and a faster degradation rate of the biodegradable polymer device. Enhance, a process that does not decrease the polymer Mn, crystallinity or orientation, and does not compromise its purity, preserved the mechanical and degradation properties of the device. In general, the higher the mechanical property of a degradable polymeric device, the slower is its degradation rate. This is obviously influenced by the shape of the device, its surface area and the porosity that may be desired or a result of the processing. 
Table 1. Mechanical and degradation properties of biodegradable polymers. Comparison with bone, ceramics and metals (Black, 1992).

\begin{tabular}{|c|c|c|c|c|c|}
\hline & $\begin{array}{l}\text { Modulus } \\
\text { (GPa) }\end{array}$ & $\begin{array}{l}\text { Strength } \\
\text { (MPa) }\end{array}$ & $\begin{array}{c}\text { Elongation } \\
\text { (\%) }\end{array}$ & $\begin{array}{c}\text { Total } \\
\text { Strength } \\
\text { loss } \\
\text { (months) }\end{array}$ & $\begin{array}{c}\text { Degradation } \\
\text { time } \\
\text { (months) }\end{array}$ \\
\hline Bone & $7-40$ & $90-120$ & & & \\
\hline \multicolumn{6}{|l|}{ Metals and Ceramics } \\
\hline $\begin{array}{l}\text { Titanium alloy } \\
\text { Stainless steel } \\
\text { Magnesium } \\
\text { Hydroxyapatite } \\
\text { Tricalcium phosphate }\end{array}$ & $\begin{array}{c}110-127 \\
180-205 \\
41-45 \\
80-110 \\
-\end{array}$ & $\begin{array}{c}900 \\
500-1000 \\
65-100 \\
500-1000 \\
154\end{array}$ & $\begin{array}{l}10-15 \\
10-40 \\
- \\
- \\
-\end{array}$ & $\begin{array}{c}\text { no } \\
\text { no } \\
<1 \\
>12 \\
1-6\end{array}$ & $\begin{array}{c}\text { no } \\
\text { no } \\
0.25 \\
>24 \\
<24\end{array}$ \\
\hline \multicolumn{6}{|l|}{ Degradable Polymers } \\
\hline $\begin{array}{l}\text { Poly(glycolic acid) (PGA) } \\
\text { Poly(L-lactic acid) (PLLA) } \\
\text { Poly(D,L-lactic-co-glycolic acid ) (PLGA) }\end{array}$ & $\begin{array}{l}7.0 \\
2.7 \\
2.0\end{array}$ & $\begin{array}{c}340-920 \\
80-500 \\
40-55\end{array}$ & $\begin{array}{l}15-20 \\
5-10 \\
3-10\end{array}$ & $\begin{array}{l}1 \\
3\end{array}$ & $\begin{array}{l}6 \text { to } 12 \\
>24 \\
1 \text { to } 12\end{array}$ \\
\hline $\begin{array}{l}\text { Poly( } \varepsilon \text {-caprolacatone) (PCL) } \\
\text { Polyurethane based on PCL and Polyethylene oxide (PEO) }\end{array}$ & $\begin{array}{c}0.4 \\
0.01-0.001\end{array}$ & $\begin{array}{c}20-40 \\
1-50\end{array}$ & $\begin{array}{l}300-500 \\
>500\end{array}$ & $\begin{array}{c}>6 \\
1 \text { to }>6\end{array}$ & $\begin{array}{c}>24 \\
6 \text { to }>24\end{array}$ \\
\hline
\end{tabular}

Regarding the mechanical properties of biodegradable devices, no significant improvements have been achieved since the development of extrusion process for the orientation and the increase of the polyester's crystallinity (Tormälä et al., 1986) (Table 2).

Processed polyesters have stiffness values in the following decreasing order; SR-PGA, SR-PLLA, moulded PLLA, moulded PGA, moulded PDS and moulded PLGA (Table 2). Carbon fibre reinforced polymers have also been developed to improve the mechanical properties of biodegradable polymeric devices, but because of the poor adhesion in-between the fibres and the polymeric matrix, rapid mechanical failure was often observed upon degradation (Zimmerman et al., 1987). Moreover, the carbon fibres did not degrade in vivo. The consequence is that most of the degradable fixation devices on the market, designed to withstand some mechanical load are SR processed polymer materials. Self-reinforcement can be described as a moulding-extrusion process and consists in polymer fibres orientation into a matrix made of the same polymer (Tormälä et al., 1986; 1987). The shaped devices can have initial strength close to metal ones (Tunc, 1991; Ashammakhi et al., 2004).

\section{Degradation}

The degradation behaviour of a degradable polymer is associated with changes in its molecular structure, its geometry and most importantly for a fixation device its mechanical properties. Wu and Ding (2005) reported the investigation of the in vitro degradation properties of poly(L-lactic-co-glycolic acid) PLGA 85:15. They suggested the division of the polyester degradation profile into 3 stages. The first stage is called "quasi-stable" which lasts as long as the measured weight, the sample shape, the mechanical properties and structural integrity remain constant. Meanwhile, the average polymer weight starts to decrease. A second stage, called "loss-of-strength" stage, begins when the device's Young Modulus decreases, while the weight loss and structural change are not yet significant.
This stage ends when the third stage called "structuredisruption" stage begins, which happens from the first significant weight loss observation until the complete material disappearance. When considering a degradable material for use as a fixation device, it is important that it retains its mechanical properties until the bone has healed (stage 1 time equivalent). Moreover, the following loss of mechanical properties should be progressive enough to allow the new bone to withstand and remodel under the increasing load (stage 2). This information, even if it is usually accepted that polyesters degrade faster in vivo than in vitro, can then be used to select a biodegradable polymer composition that may fit the desired degradation pattern for the medical device. Although, the degradation behaviour of a polyester device depends strongly on the polymer Mn, its crystallinity, purity as well as the presence of reinforcing structure and its orientation, introduced during the processing of the device.

\section{Degradable vs. non-degradable devices}

Mechanical properties. Compared to metallic devices, polymeric devices are less brittle because of a lower modulus of elasticity (Table 1), but they undergo more creep and stress relaxation. This causes loosening, which can be up to $20 \%$ of a degradable polymeric screw's initial holding force, causing greater fracture mobility (Claes, 1992). A degradable device such as a PLLA plate with equivalent design and strength of a titanium plate is more bulky (e.g., $2 \mathrm{~mm}$ PLLA plate equivalent $1.5 \mathrm{~mm} \mathrm{~K}$-wires) (Waris et al., 2002). Having a bulky device may be detrimental in many ways but, most notably, it may be difficult to avoid the autocatalytic degradation process observed in polyester materials and the associated drawbacks. Moreover, large devices can be unsuitable for many specific trauma surgeries like in hand surgery, where low profile and gliding are essential.

Biocompatibility. Historically there was deep concern concerning the biocompatibility of polymeric devices when compared to metallic implants and the potential for aseptic 
Table 2. Tensile strengths of PLLA and PGA devices prepared using different process (Simon et al., 1998; Tormälä et al., 1986).

\begin{tabular}{lll}
\hline Process & \multicolumn{2}{l}{ Shear Strength (MPa) } \\
& PLLA & PGA \\
\hline Injection-moulding & $80-200$ & $80-110$ \\
Solid state extrusion & $100-350$ & $200-250$ \\
Self reinforced (SR) & 300 & 200 \\
\hline
\end{tabular}

inflammation from wear debris generated during the implant degradation. Complications have been reported including sterile sinus tract formation, osteolysis, synovitis and hypertrophic fibrous encapsulation (Waris et al., 2002). Depending on the poly ( $\alpha$-hydroxyacid) homopolymer and site of implantation, adverse tissue reactions due to inflammatory responses, nonunion, etc have been observed with a higher occurrence with the PGA than the PLLA devices (Bostman and Pihlajamaki, 2000). This was attributed to their respective degradation rate and the process of by-products absorption (Taylor et al., 1994). This is to a large extent due to the first generation of polymers used; PGA and PLLA, their crystallinity, purity and processing.

A worthy illustration is the PLLA screw for bone fixation developed in the early $80 \mathrm{~s}$. Due to the size and shape of the screws, bulk degradation occurred with the build up of degradation products, the burst release of the acid by-products and the $\mathrm{pH}$ decrease in close vicinity of the screw. The consequence was that bone formation was affected. Moreover, with more than 10 years of surgery history, it has been shown that residual crystalline particle wear debris was often present in the area of the screw and could also affect the normal bone healing (Simon et al., 1997; Dunne et al., 2000).

Degradation properties. Nowadays, the development of PLGA and other co-polymers that are not initially crystalline, and do not present a burst release upon degradation, have minimized the foreign body reaction associated with crystalline polyesters. However, one of the major difficulties is the follow up of the degradable devices which may take as much as 18 years to degrade fully in living tissue and enhance the loss of their major advantage (e.g. no implant removal necessary), compared to metallic implants (Pistner et al., 1993). Hence, there is a need for polymers that would degrade completely without leaving traces within 6 to 18 months, while implants from these polymers would maintain their mechanical functionality over the entire period necessary for bone fracture healing (e.g. 6 months). Among the promising polymers, biodegradable amorphous ter-polymers based on randomly distributed repeating units of lactides, glycolides and caprolactones have been synthesized and their degradation profile characterized (Glarner and Gogolewski, 2007). The molecular chain irregularity of these polymers as for the PLGA co-polymer, affects their crystallinity and facilitates the diffusion of molecules in and out of the polymers, and therefore their homogeneous degradation (Fig. 3).
The composition of the ter-polymers produced allows some control over the degradation stages of the polymers. The stage I (constant mechanical properties) can be varied from less than a week to 20 weeks, followed by a steady decrease of mechanical properties (decrease of bending stress in 8 to 20 weeks) without dramatic mechanical failure and bulk degradation compared to PLLA (Figure 2) (Glarner and Gogolewski, 2007).

\section{Biodegradable Osteosynthesis Devices}

Degradable polymers are principally used to replace metals in fixations that are under very low load and when degradation and material integration are highly valuable for the patient's outcome (Fig. 4).

For craniomaxillofacial CMF surgeries, titanium implants are not without drawbacks. Removal of the implant is necessary in $12 \%$ of patients because of thermal conductivity, allergic hypersensitivity, chemical carcinogenesis, infection, etc (Matthew and Frame, 1999). The biodegradable implants overcome the metal implants' pitfalls and are frequently used in CMF surgery, and markedly in paediatric CMF surgeries (Fig. 4). Biodegradable devices are also utilized to overcome the limitation of the non-degradable devices in others areas of reparative medicine such as foot, ankle, elbow, hand and wrist fracture treatment and spinal fusion surgery (Simon et al., 1997b). An example is the radiolucent PLDLLA cages used in interbody fusion techniques (Wuisman and Smit, 2006). PLDLLA cages implanted in a small number of patients offered a promising outcome (Kuklo et al., 2004). Although, in this specific case, fast degradation or even complete degradation may not be advantageous, and non-degradable polymers such as poly(etheretherketone) may be more suitable. A common use of degradable interference screws is the anterior cruciate ligament reconstruction. A recent study has also shown the potential of these screws as an alternative to titanium screws for the fixation of autologous bone grafts in dental implants (Raghoebar et al., 2006). On the whole, for more than two decades, biodegradable osteosynthesis devices have been used in many surgeries. Still, there are some important issues that are being discussed such as the infection risk associated with the biodegradable devices, their mechanical stability and the real gain in terms of healing success when compared to metallic devices.

\section{Infection}

The susceptibility of degradable polymeric devices to bacteria infection and biofilm formation is more complex than on metallic implants. Polymers of different composition have a potentially distinct response. Processes, sterilization and time of implantation can also modify the devices' interaction with bacteria. PLLA surfaces have been shown to be more prone to bacterial infection than titanium surface with an infection rate of $50 \%$, ten times lower for the polylactide when compared to metal (Hauke et al., 1996; Schlegel and Perren, 2006). The resistance to infection of two degradable polymeric devices (PLLA and PLDLLA) has been compared in an animal model. Both 

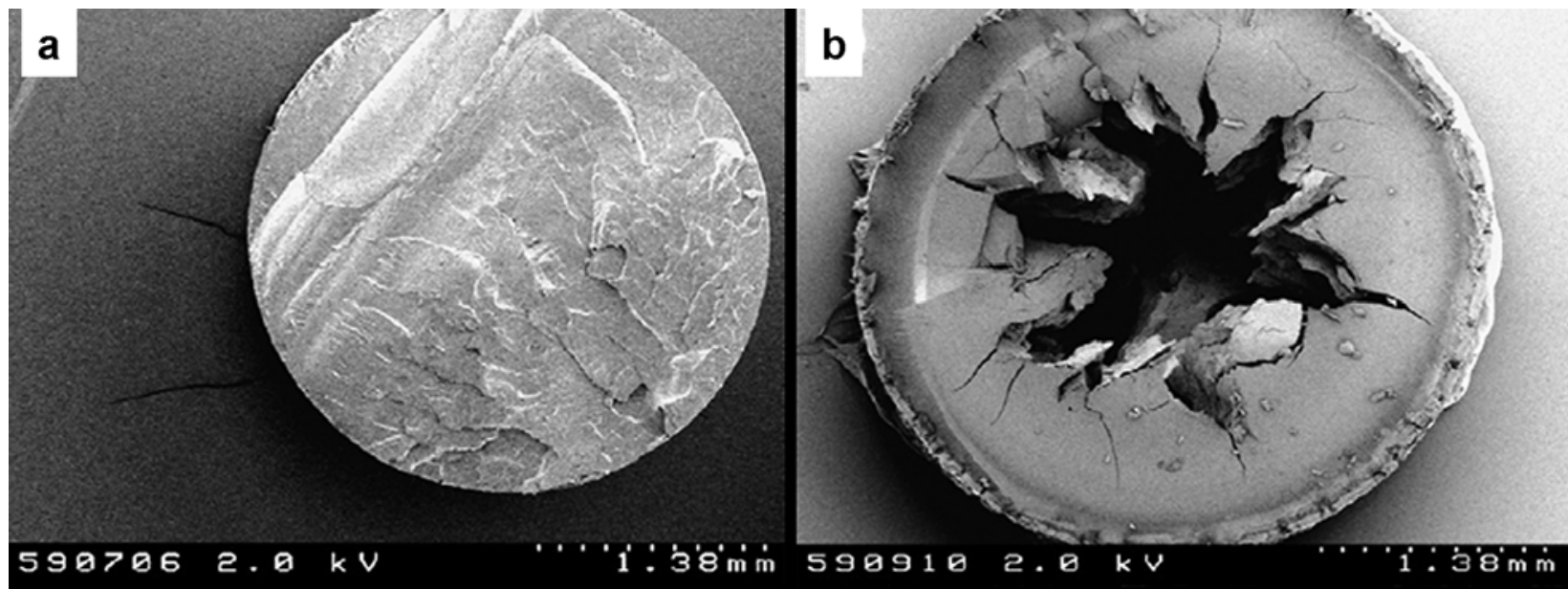

$1: 38^{\circ} \mathrm{mm}$

$i: 3 \mathbf{s}^{\prime} \mathrm{m}$
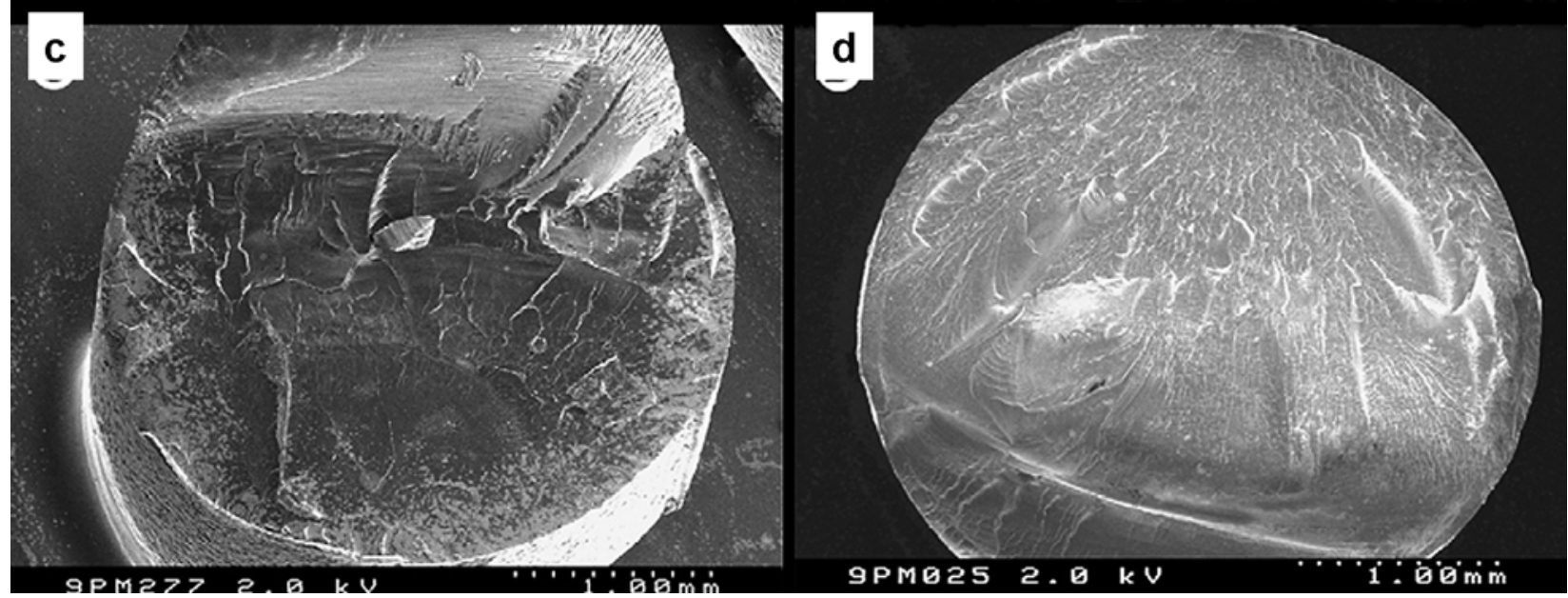

Figure 3. Scanning electron microscopy images of samples illustrating in vitro degradation pattern of the poly(Llactide) and terpolymer at 0 and 24 weeks in a simulated body fluid. Micrographs a and b for poly(L-lactide), and $\mathrm{c}$ and $\mathrm{d}$ for terpolymer (courtesy of Richards RG and Glarner M).

polymer compositions are equally resistant to local infection and, when the infection is created, the degradation products of both polymeric devices do not affect the established infection (Mainil-Varlet et al., 2001). Also, studies have shown that, in vitro, Staphylococcus aureus adhesion was significantly reduced on PLLA compared to metal whereas the inverse has been observed for Staphylococcus epidermis (Barth et al., 1989). Thus, it can then be summarized that the poly( $\alpha$-hydroxyacid $)$ compositions do not affect significantly bacterial adhesion and infection. Degradable polymers are colonized by bacteria, similarly to non-degradable polymer surfaces, with probably a higher risk of infection than on metal implants - depending on the bacterial strain. Finally, the sterilization of a polymeric implant is more complicated than a metal one and can only be performed once. Common dry heat and autoclaving sterilizations cannot be carried out as they significantly modify the biodegradable device's specifications. Typically, ethylene oxide and radiations are employed to minimize degradation during polymer device sterilization. In early studies, these difficulties with the necessity of a careful storage to avoid early degradation may have been the cause of infection related to the release of by-products, screw loosening, etc. (Middleton and Tipton, 2000).

\section{Stability}

Early studies on the clinical stability of osteosynthesis fixations found no significant difference between titanium and degradable devices (Matthew and Frame, 1999). It has been reported that higher breakage of biodegradable screws and plates has occurred essentially due to the more demanding handling of the biodegradable devices. The rate of breakage decreases after the surgeons become familiar with the biodegradable implants, which tend to be more bulky and have poorer handling than the titanium ones (Eppley et al., 2004). Nonetheless, loosening of biodegradable polymeric fixations often occurs due to creep. A recently reported commercially available technique that surmounts this problem is based on an ultrasound device melting and welding of the biodegradable device (pin) into bone tissue. This method improves the stability of the biodegradable device and reduces time for the fixing of the device, when compared to conventional degradable screws with no thread cutting required (Eckelt et al., 2007). The drawback of such a technique is that the necessary melting of the degradable pin makes it difficult to obtain a high strength material. Thus, the accurate control of the device degradation and mechanical properties may be difficult. In the meantime, in another study comparing PLGA and titanium Le Fort I 


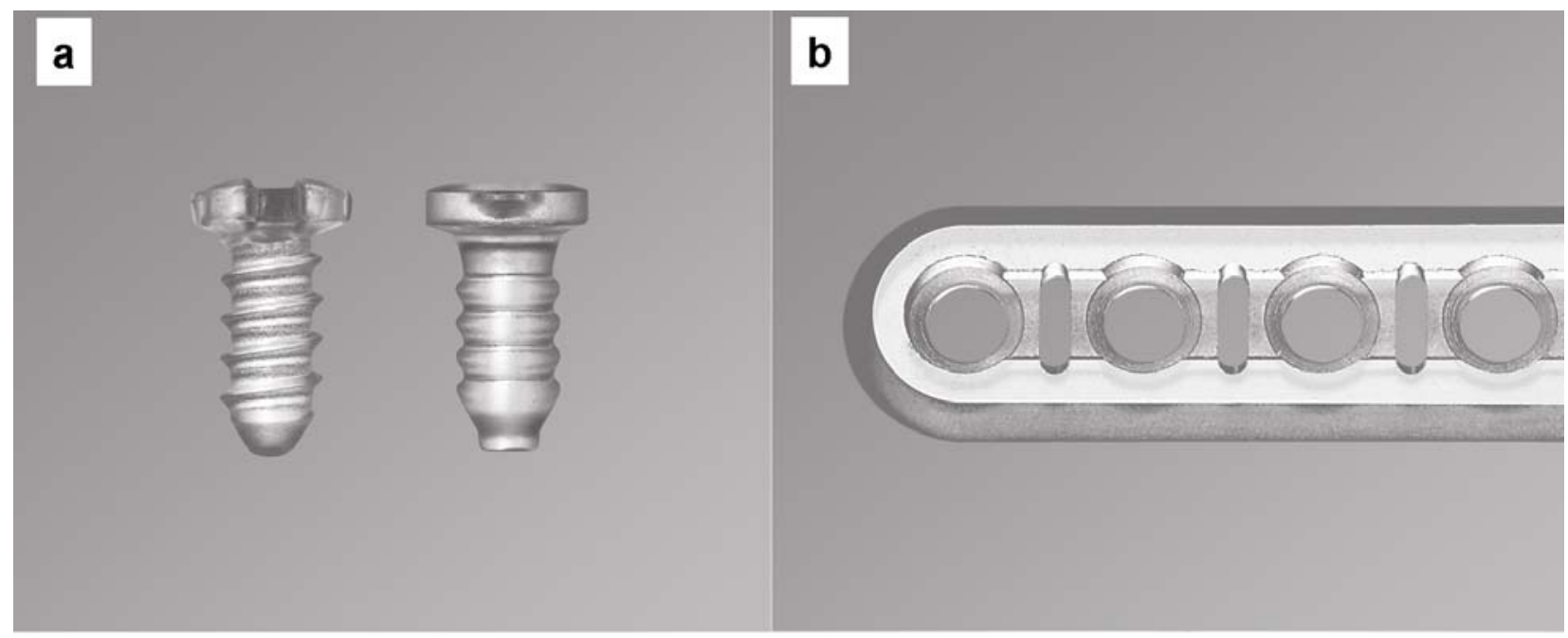

Figure 4. Resorbable CMF screw and tack (a), and plate (b) RapidSorb Resorbable Fixation System by Synthes $\mathrm{GmbH}$.

osteosynthesis miniplate, a slight significant change in maxillary position has been observed as measured by cephalometric analysis of tantalum reference implants (Norholt et al., 2004). This indicated a lower stability of the fracture when using PLGA device. However, this was not noticeable clinically and the outcome of the surgery was satisfactory for all patients. In conclusion, it seems that biodegradable devices can function as well as metallic devices in terms of implant stability.

\section{Biodegradable vs. non-biodegradable devices}

Controlled studies on the advantages of biodegradable versus metallic or non-degradable polymer osteosynthesis devices have been scarce until recent years. For example, biodegradable devices have the obvious advantage in hand surgery for the avoidance of soft tissue adhesion on implants, but only a few studies are available and none investigate the real benefit of degradable fixation against more traditional fixations in a controlled fashion (Hughes, 2006). Retrospective studies have been reported, but they are often of poor value as they usually do not generate significant and conclusive answers to questions such as the possible higher risks of infection and failure using biodegradable devices. In fact, it is only this last decade that controlled comparison studies between metal and biodegradable devices have became available, and nearly exclusively for CMF surgery (Cheung et al., 2004). Cheung et al. (2004) published one of the first randomized controlled trials concerning the comparison of biodegradable and titanium fixations. In this controlled comparison study, no significant difference in the rate of infection $1.53 \%$ and $1.83 \%$ respectively for titanium and SR-PLLA fixations, is observed. The outcome of a randomized study of the treatment of displaced radial head fractures with PLDLLA pins and metal implants has also evidenced that complication rates and clinical outcomes were comparable when using biodegradable and nondegradable implants (Helling et al., 2006). In a study, two biodegradable miniplate systems are compared in sagittal split osteotomies with major bone movement (Landes and Kriener, 2003). No significant difference between the two systems is observed and relatively good stability is achieved when using two biodegradable plates. Although the authors wished for more rigid and smaller degradable implants, because of breakage upon implantation and the inadequate implant dimension. This is in accordance with a compilation of 1883 paediatric craniofacial surgery cases that show postoperative infection lower than $1 \%$ after a primary surgery for degradable implants (Eppley et al., 2004). Similar randomized trial performed in other areas of reparative medicine, namely in wrist fractures, reports comparable results between the two groups, PLGA and titanium implants, in term of re-operation and wrist functionality (Van Manen et al., 2008).

Finally, a recent review by Jainandunsing et al. (2005) compiles the results of thirty-one published randomized and quasi-randomized controlled trials published in the literature since 1988, comparing difference in outcome between biodegradable and metal fixation devices for fixation of bone fractures or re-attachment of soft tissue to bone in adult patients. The authors conclude that there are enough randomized controlled trials that indicate that biodegradable implants are as good as metal implants when considering clinical outcome, complication rate and infections. However, they point out the need for higher quality reported trials with better defined treated injuries, the need to include cost-effective analysis, and the need for sufficient and longer follow up on patients.

\section{Biodegradable vs. biodegradable devices}

A number of osteosynthesis devices made of different biodegradable polymer composition are commercially available. Only a few studies have compared the effect of the biodegradable polymers on the outcome of a reparative surgery performed. One of the first, compared implants prepared from poly(L-lactic-co-D,L-lactic acid) PLDLLA $80: 20$ and 70:30 composition and used for surgery of 23 human patients with scaphoid failure of broken bones 
(Akmaz et al., 2004). For fixation of bone fractures in regions of low load, the investigation shows that the higher chemical strength and loading capacity of PLDLLA 80:20, due to higher crystallinity is advantageous for long term implantation. Also, long-term follow up has not been reported. Landes et al. (2006) report a 5-years experience with more than 400 implantations of biodegradable plate for osteosynthesis made of poly(L-lactic-co-glycolic acid) PLGA 85:15 and PLDLLA 70:30. Both devices degrade after 12 months and 24 months of implantation respectively for PLGA 85:15 and PLDLLA 70:30, leaving crystalline polymeric particles that are not detrimental to the bone formation in this specific application. Foreign body reaction is observed for $6 \%$ of the patients 3 to 4 months after the surgery without significant difference in between the two resorbable plates. Commercial devices made of glycolic and trimethyl carbonate monomers have also shown to have better flexibility and slower degradation rate than pure PGA ( 7 months) and to be suitable as interference screws for bone-tendon graft fixation (Middleton and Tipton, 2000). Wittwer et al. (2006) compare three different biodegradable osteosynthesis materials and titanium devices, and show no significant difference in the fracture healing and the postoperative complications. These reports seem to point out that there are no clear benefits in using one or another biodegradable polyester for osteosynthesis. The relatively conservative approach taken for the design of the biodegradable devices is probably the explanation for this observation. In fact, the commercialized degradable polymeric devices are prepared from a small number of FDA approved polyesters, with similar degradation pathway and biocompatibility. Moreover, to avoid device mechanical failure the degradation time of the polymers considered is often much longer than the time of the biological tissue healing (Table 1). The consequence is that the device stays longer in the body than its useful lifetime and it is likely that some of the theoretical advantages: the avoidance of bone necrosis and the mechanical stimulation of the newly formed bone upon the device degradation for example, are lost. Without a doubt, there is the need to improve the degradation profile of the degradable polymers used for osteosynthesis in order to match more closely the bone healing process.

\section{New trends for biodegradable devices}

Polymer coating. An important issue in bone tissue surgery is to minimize the risk of infection. Usually, antibiotics are the main tools to fight infection. A valuable method to avoid infection is to reduce bacterial adhesion on implant by the control of the fixation surfaces topography and chemistry (Harris and Richards, 2006). The use of degradable polymers has been proposed for the coating of titanium plates and the local release of loaded antibiotic and antiseptic in a controlled fashion. Coating made of PLLA decreases significantly the rate of infection in vivo due to the degradation of the antibacterial coating and the release of drugs (Kalicke et al., 2006). However, failure or peeling of the coating could potentially be detrimental to bone repair. Nonetheless, antibiotic-coated intramedullar nails implanted in eight patients with open tibia fractures have been shown to be effective in preventing infection (Schmidmaier et al., 2006). A step further would be a fully degradable device with controlled surface topography and chemistry and with an antibiotic load to decrease infection. The optimization complexity of the material antonymic properties (i.e. mechanical, degradation and loaded molecules release) is likely to be the source for the lack of such commercial device yet.

Composite devices. The shift of focus on bone repair evolution from a purely mechanical stability viewpoint (conventional stable fixation) to a more biologically orientated approach (avoidance of necrosis, infection, biomechanical, biochemical and biological stimulations) has enforced the necessity of new degradable materials (Perren, 2002). In a recent study Uhtoff et al. (2006) have developed polylactide inserts in a metal plate fixation device. The purpose of these PLLA inserts that fit between the hard plate and the screw is to allow some micro-motion limited to the axial direction and stimulate bone osteogenesis while avoiding bone necrosis. Also, the authors reported the failure of the insert in an animal study and the inadequacy of the material used; this is an interesting approach toward the development of new devices for osteosynthesis. Such devices could authorize some sway on the mechanical load applied to the healing bone, while keeping the stability of the fixation. Biodegradable polyurethanes could be an interesting alternative to PLLA because of their elastomeric property and resilience under cyclic load. Although in this design, the fixation may have to be explanted and the benefit of fully degradable device is lost.

Bioactive devices. An emerging area where biodegradable polymers are currently unavoidable is in cell guidance and tissue engineering constructs for bone tissue regeneration. These approaches take advantage of the cells' adhesion and spreading over polymer surfaces and the versatile processing properties of polymer to regenerate damaged living tissue. Biodegradable film surfaces for guided tissue regeneration have been used for more than 15 years in periodontal and craniofacial surgeries (Eickholz et al., 2006). PLDLLA membranes combined with a bone filler or not, and coupled with an osteosynthesis device have shown to be a promising solution to speed up the healing of large bone defects (Gugala and Gogolewski, 1999). Three-dimensional degradable structures have also been developed as bone substitutes. New bone formation and growth occur into the porous structure, which degrades over time, leaving a new regenerated bone tissue. Further improvement of the bone healing process has been attained by combining bio-molecules such as growth factors (e.g. bone morphogenic proteins) with biodegradable devices (Jain et al., 1998). The last move forward has been the creation of tissue engineering constructs composed of a biodegradable porous structure, a biological stimulus such as growth factors and a biological component such as autologous cells. In this approach, the material, a support for bone regeneration, has a design which is the antithesis of the actual osteosynthesis fixations. 


\section{Conclusion}

There is not yet a biodegradable fixation available for long bone support such as the femur. However, degradable polymeric devices for osteosynthesis are shown some success in low or mild load bearing applications. The choice between degradable and non-degradable devices should be carefully weighed and depends on many factors such as the patient condition, the type of fracture, etc. The lack of carefully controlled randomized prospective trials that document their efficacy in treating particular fracture patterns and to show their superiority is still an issue. The current generation of biodegradable implants for bone repair, made of polymers and with designs copied from metal implants, originates from the concept that devices should be supportive and "inert" substitute to bone tissue. Meanwhile, the last advances in the field of regenerative medicine have shown that with the better understanding of the biological mechanisms and factors that influence living tissue regeneration, these devices may be "active" rather than "passive", leading to hope for new therapies. The next generation of biodegradable implants will probably see the implementation of the recently gained knowledge in cells therapy, with a better control of the spatial and temporal interfaces between the material and the surrounding biological tissue.

\section{Acknowledgements}

D. Eglin would like to thank Dr R.G. Richards and Dr S. Poulsson for their helpful suggestions and Dr S. Beck for kindly providing the Synthes $\mathrm{GmbH}$ resorbable device pictures.

\section{References}

Akmaz I, Kiral A, Pehlivan O, Mahirogullan M, Solakoglu C, Rodop O (2004) Biodegradable implants in the treatment of scaphoid nonunions. Int Orthop 28: 261266.

Andriano KP, Pohjonen T, Tormal P (1994) Processing and characterization of absorbable polylactide polymers for use in surgical implants. J Appl Biomat 5: 133-140.

Ashammakhi N, Gonzalez AM, Tormala P, Jackson IT (2004) New resorbable bone fixation. Biomaterials in craniomaxillofacial surgery: present and future. Eur J Plast Surg. 26: 383-390.

Barth E, Myrvik QM, Wagner W, Gristina AG (1989) In vitro and in vivo comparative colonization of Staphylococcus aureus and Staphylococcus epidermidis on orthopaedic implant materials. Biomaterials 10: 325328.

Black J (1992) Biological Performance of Materials: Fundamentals of Biocompatibility. $2^{\text {nd }}$ ed. Marcel Dekker, New York.

Bohner M (2000) Calcium orthophosphates in medicine: from ceramics to calcium phosphate cements. Injury 31: 37-47.
Bonzani IC, George JH, Stevens MM (2006) Novel materials for bone and cartilage regeneration. Curr Opin Chem Biol. 10: 568-575.

Bostman OM, Pihlajamaki HK (2000) Adverse tissue reactions to bioabsorbable fixation devices. Clin Orthop Rel Res 371: 216-227.

Chandra R, Rustgi R (1998) Biodegradable polymers. Progr Polym Sci. 23: 1273-1335.

Cheung LK, Chow LK, Chiu WK (2004) A randomized controlled trial of resorbable versus titanium fixation for orthognathic surgery. Oral Surg Oral Med Oral Pathol Radiol Endod 98: 386-397.

Claes LE (1992) Mechanical characterization of biodegradable implants. Clin Mater. 26: 1553-1567.

Dunne M, Corrigan OI, Ramtoola Z (2000) Influence of particle size and dissolution conditions on the degradation properties of polylactide-co-glycolide particles. Biomaterials 21: 1659-1668.

Eckelt U, Nitsche M, Muller A, Pilling E, Pinzer T, Roesner D (2007) Ultrasound aided pin fixation of biodegradable osteosynthetic materials in cranioplasty for infants with craniosynostosis. J Cranio-Maxillofac Surg 35: 218-221.

Eickholz P, Pretzl B, Holle R, Kim TS (2006) Longterm results of guided tissue regeneration therapy with nonresorbable and bioresorbable barriers. III. Class II furcations after 10 years. J Periodontol. 77: 88-94.

Eppley BL, Morales L, Wood R, Pensler J, Goldstein J, Havlik RJ, Habal M, Losken A, Williams JK, Burstein F, Rozzelle AA, Sadove AM (2004) Resorbable PLLAPGA plate and screw fixation in pediatric craniofacial surgery:clinical experience in 1883 patients. Plast Reconstr Surg 114: 850-856.

Glarner M, Gogolewski S (2007) Degradation and calcification in vitro of new bioresorbable terpolymers of lactides with an improved degradation pattern. Polym Deg Stab 92: 310-316.

Gogolewski S (1997) Biomedical polyurethanes. In: Desk Reference of Functional Polymers. Syntheses and Applications (Arshady R, ed), American Chemical Society, 677-698.

Gogolewski S (2000) Bioresorbable polymers in trauma and bone surgery. Injury 31: 28-32.

Gogolewski S, Gorna K (2007) Biodegradable polyurethane cancellous bone graft substitutes in the treatment of iliac crest defects. J Biomed Mater Res Part A. 80: 94-101.

Gugala Z, Gogolewski S (1999) Regeneration of segmental diaphyseal defects in the sheep tibiae using resorbable polymeric membranes. A preliminary study. J Orthop Trauma 13: 187-195.

Harris LG, Richards RG (2006) Staphylococci and implant surfaces: a review. Injury 37: S3-S14.

Hauke C, Schlegel U, Melcher GA, Printzen G, Perren SM (1996) Einfluss des implantatmaterials auf die lokale infektresistenz bei der tibiamarknagelung. Eine experimentelle vergleichsstudie amd kaninchen mit marknageln aus rostfreiem stahl und reintitan. Swiss Surg 45: $52-58$. 
Helling H-J, Prokop A, Schmid HU, Nogel M, Lilienthal J, Rehm KE, Ludwigsburg K, Germany H (2006) Biodegradable implants versus standard metal fixation for displaced radial head fractures. A prospective, randomized, multicenter study. J Shoulder Elbow Surg 15: 479-485.

Hughes TB (2006) Bioresorbable Implants In the treatment of hand fractures. An update. Clin Orthop Rel Res. 445: 169-174.

Jain R, Shah NH, Malick AW, Rhodes CT (1998) Controlled drug delivery by biodegradable poly(ester) devices: different preparative approaches. Drug Dev Ind Pharm. 24: 703-727.

Jainandunsing JS, Van Der Elst M, Van Der Werken CC (2005) Bioresorbable fixation devices for musculoskeletal injuries in adults. Cochrane Database Syst Rev 2: 1-43.

Kalicke T, Schierholz J, Schlegel U, Frangen TM, Koller M, Printzen G, Seybold D, Klockner S, Muhr G, Arens S (2006) Effect on infection resistance of a local antiseptic and antibiotic coating on osteosynthesis implants: An in vitro and in vivo study. J Orthop Res. 24: 1622-1640.

Kuklo TR, Rosner MK, Polly DW (2004) Computerized tomography evaluation of a resorbable implant after transforaminal lumbar interbody fusion. Neurosurg Focus 16: E10.

Landes CA, Kriener S (2003) Resorbable plate osteosynthesis of sagittal split osteotomies with major bone movement. Plast Reconstr Surg. 111: 1828-1840.

Landes CA, Ballon A, Roth C (2006) Maxillary and mandibular osteosyntheses with PLGA and P(L/DL)LA implants: A 5-year in patient biocompatibility and degradation experience. Plast Reconstr Surg. 117: 2347 2360 .

LeGeros RZ (2002) Properties of osteoconductive biomaterials: calcium phosphates. Clin Orthop Relat Res. 395: 81-98.

Li S, Garreau H, Vert M (1990) Structure-property relationships in the case of the degradation of massive poly $(\alpha$-hydroxyacids) in aqueous media. Part 3: Influence of the morphology of the poly(L-lactic acid). J Mat Sci Mat Med. 1: 198-206.

Li S (1999) Hydrolytic degradation characteristics of aliphatic polyesters derived from lactic and glycolic acids. J Biomed Mater Res. Appl Biomat. 48: 342-353.

Mainil-Varlet P, Hauke C, Maquet V, Printzen G, Arens S, Schaffner T, Jerome R, Perren S, Schlegel U (2001) Polylactide implants and bacterial contamination: an animal study. J Biomed Mater Res 54: 335-343.

Matthew IR, Frame JW (1999) Policy of consultant oral and maxillofacial surgeons toward removal of miniplate components after jaw fracture fixation: pilot study. Brit J Oral Maxillofac Surg. 37: 110-112.

Middleton JC, Tipton AJ (2000) Synthetic biodegradable polymers as orthopedic devices. Biomaterials 21: 2335-2346.

Norholt SE, Pedersen TK, Jensen J (2004) Le Fort I miniplate osteosynthesis: A randomized, prospective study comparing resorbable PLLA/PGA with titanium. Int J Oral Maxillofac Surg 33: 245-252.
Perren SM (2002) Evolution of the internal fixation of ling bone fractures. J Bone Joint Surg 84B: 193-1110.

Pistner H, Bendix DR, Muhling J, Reuther JF (1993) Poly(l-lactide): a long-term degradation study in vivo. Part III. Analytical characterization. Biomaterials 14: 291-298.

Pitt CG, Gratzl MM, Kimmel GL, Surles J, Schindler A (1981) Aliphatic polyesters II: The degradation of poly(DL-lactide), poly( $\varepsilon$-caprolactone), and their copolymers in vivo. J Biomat. 2: 215-220.

Pitt CG (1992) Non-microbial degradation of polyesters. In: Biodegradable Polymers and Plastics. Mechanisms and Modifications (Vert M, Feijen J, Albertsson A, Scott G, Chiellini E, eds), Royal Soc Chem, Cambridge, pp 7-17.

Raghoebar GM, Liem RSB, Bos RRM, Van Der Wal JE, Vissink A (2006) Resorbable screws for fixation of autologous bone grafts. Clin Orl Impl Res. 17: 288-293.

Rockwood DN, Woodhouse KA, Fromstein JD, Chase DB, Rabolt JF (2007) Characterization of biodegradable polyurethane microfibers for tissue engineering. J Biomed Sci Polym Ed.18: 743-758.

Schmidmaier G, Lucke M, Wildemann B, Haas NP, Raschke M (2006) Prophylaxis and treatment of implantrelated infections by antibiotic-coated implants: a review. Injury 37: 105-112.

Schlegel U, Perren SM (2006) Surgical aspects of infection involving osteosynthesis implants: implant design and resistance to local infection. Injury 37: 67-73.

Simon JA, Ricci JL, Di Cesare PE (1997) Bioresorbable fracture fixation in orthopedics: a comprehensive review. Part II. Clinical studies. Amer J Orthop. 26: 754-762.

Simon JA, Ricci JL, Di Cesare PE (1998) Bioresorbable fracture fixation in orthopedics: a comprehensive review. Part I. Basic science and preclinical studies. Amer J Orthop. 26: 665-671.

Staiger MO, Pietak AM, Huadmai J, Dias G (2006) Magnesium and its alloys as orthopedic biomaterials: A review. Biomaterials 27: 1728-1734.

Taylor MS, Daniels AV, Andriano KP, Heller J (1994) Six bioabsorbable polymers: In vitro acute toxicity of accumulated degradation products. J Appl Biomat 5: 151157.

Tormälä P, Laiho J, Helevirta P, Rokkanen P, Vainionpää S, Böstman O, Kilpikari J (1986) Resorbable surgical devices. In: Proceedings of the Vth International Conference on Polymers in Medicine and Surgery, PIMS, Leeuwenhorst Congress Center, The Netherlands, pp 16/ 1-16/6.

Tormälä P, Vainionpää S, Kilpikari J, Rokkanen P (1987) The effect of fibre reinforcement and gold plating on the flexural and tensile strength of PGA/PLA copolymer materials in vitro. Biomaterials 8: 42-45.

Tunc DC (1991) Body-absorbable osteosynthesis devices. Clin Mater. 8 119-123.

Uhthoff HK, Poitras P, Backman DS (2006) Internal plate fixation of fractures: short history and recent developments. J Orthop Sci. 11: 118-126.

Van der Elst M, Patka P, Van der Werken C (2000) Resorbierbare Implantate Fur Frakturfixierungen. Unfallchirurg 103: 178-182. 
Van Manen CJ, Dekker ML, Van Eerten PV, Rhemrev SJ, Van Olden GDJ, Van der Elst M (2008) Bio-resorbable versus metal implants in wrist fractures. Arch Orthop Trauma Surg 128:1413-1417.

Vert M, Li MS, Spenlehauer G, Guerin P (1992) Bioresorbability and biocompatibility of aliphatic polyesters. J Mater Sci 3: 432-446.

Vert M (2005) Aliphatic polyesters: Great degradable polymers that cannot do everything. Biomacromolecules 6: $538-546$.

Von Burkersroda F, Schedl L, Gopferich A (2002) Why degradable polymers undergo surface erosion or bulk erosion. Biomaterials 23: 4221-4231.

Waris E, Ashammakhi, Raatikainen T, Tormala P, Santavirta S, Konttinen YT (2002) Self-reinforced bioabsorbable versus metallic fixation systems for metacarpal and phalangeal fractures: a biomechanical study. J Hand Surg 27: 902-909.
Williams D (1981) Enzymatic degradation of polylactic acid. Eng in Med 10: 5-7.

Wittwer G, Adeyemo WL, Yerit K, Voracek M, Turhani D, Watzinger F, Enislidis G (2006) Complications after zygoma fracture fixation: is there a difference between biodegradable materials and how do they compare with titanium osteosynthesis? Oral Surg Oral Med Oral Pathol Oral Radiol Endod 101: 419-25.

Wu L, Ding J (2005) Effects of porosity and pore size on in vitro degradation of three-dimensional porous poly(D,L-lactide-Co-glycolide) scaffolds for tissue engineering. J Biomed Mater Res Part A. 75: 767-777.

Wuisman PIJ, Smit TH (2006) Bioresorbable polymers: Heading for a new generation of spinal cages. Eur Spine J 15: 133-148.

Zimmerman M, Parsons JR, Alexander H (1987) The design and analysis of a laminated partially degradable composite bone plate for fracture fixation. J Biomed Mater Res 21: 345-361. 